\title{
Invariants for Smooth Conjugacy of Hyperbolic Dynamical Systems, III
}

\author{
José Manuel Marco` and Roberto Moriyón` \\ Universidad Autónoma de Madrid
}

\begin{abstract}
We give a characterization of Anosov diffeomorphisms smoothly conjugated to a toral automorphism in dimension two in term of the Lyapunov exponents of periodic points. We also give necessary and sufficient conditions for the regularity of solutions of the vector cohomology equations associated to an Anosov flow in three dimensions. This allows us to prove a corresponding conjugation theorem.
\end{abstract}

\section{Introduction}

In this paper we continue the study of smooth conjugacy problems for general Anosov systems, initiated in [MM, L1]. We are concerned with two questions. i) Given two Anosov diffeomorphisms on the 2-dimensional torus $T^{2}$, when are they $C^{\infty}$ conjugated? (This question is solved in [L1] when they are close enough in the $C^{1}$ topology). ii) Give necessary and sufficient conditions for the existence of a $C^{\infty}$ conjugation between vector fields on a 3-dimensional compact manifold that generate Anosov flows (no result of this kind was previously known).

A motivation for these questions comes from inverse spectral problems in riemannian geometry (see $[\mathrm{GK}]$ ), where one tries to construct isometries by smoothly conjugating the corresponding geodesic flows. Collet, Epstein and Gallavotti, [CEG], study them in relation with integrability properties of hamiltonian systems in a general sense. On the other hand, smooth conjugation problems are interesting by themselves, and they have given rise to important and beautiful Mathematics, as in $[\mathrm{H}]$.

In the case of diffeomorphisms of the torus, we probe that a $C^{\infty}$ Anosov diffeomorphism is $C^{\infty}$ conjugated to a toral automorphism if and only if the Lyapunov exponents of all periodic orbits are the same. In the second case we show that the periods and the Lyapunov exponents of periodic orbits are a complete set of invariants for the smooth conjugacy of one-parameter families of Anosov flows to

* Supported by CAICyT grant \# 397/84 
one of them. This clarifies the role of Lyapunov exponents in this context, which was not clear in view of the example in [CEG, Appendix E].

Our approach in the case of flows is close to the original approach of Moser (see [Ma, Mo]) to the proof of Anosov's stability theorem, and to the study in [LMM] of similar problems for hamiltonian flows. The conjugation problem is reduced to a linear problem for vector fields. We find necessary and sufficient conditions for the regularity of the solutions of vector cohomological equations $\left(L_{X} Y=Z\right.$, or $f_{*} Y-Y=Z$ in the case of maps) that are interesting by themselves.

The main open problems related to these questions are the extension to higher dimensions, and proving similar results for isolated flows instead of parametric families of them. Finally, the study of the real analytic case is something that is missing in the whole theory.

\section{Global Smooth Conjugacy of Toral Diffeomorphisms}

In this section we prove that the Lyapunov exponents of periodic points characterize toral automorphisms up to smooth conjugacy, within the class of Anosov diffeomorphisms on $T^{2}$. We also prove that the resulting conjugation is globally canonical if one of the diffeomorphisms is the composition of the other with a globally canonical map.

By a globally canonical map we mean an area preserving map on $T^{2}$ with vanishing Calabi invariant in the sense of $[C, B]$. See $[M M]$ for a definition of the Lyapunov exponents of a periodic point of a diffeomorphism.

Theorem 1. Letf: $T^{2} \rightarrow T^{2}$ be a $C^{\infty}$ Anosov diffeomorphism. Then is $C^{\infty}$ conjugate to a total automorphism if and only if the Lyapunov exponents of all periodic orbits are the same. If $f$ is real analytic, the conjugation is also real analytic. If $f$ leaves the canonical volume form invariant, so does the conjugation. If moreover $f$ is the composition of its corresponding toral automorphism with a globally canonical map, then the conjugation is globally canonical.

Proof. If $f$ is $C^{1}$ conjugate to a toral automorphism defined by a unimodular matrix $A$, it is obvious that the Lyapunov exponents of all periodic points are the logarithms of the absolute values of the eigenvalues of the matrix $A$. We shall assume now that the Lyapunov exponents are independent of the point, and we shall see that $f$ is $C^{2+\delta}$ conjugate to a toral automorphism.

Let $A$ be the $2 \times 2$ integer unimodular matrix obtained by transposition of the matrix of $f^{\#}: H^{1}(M, \mathbb{R}) \rightarrow H^{1}(M, \mathbb{R})$ in the canonical basis. It is known (see $[\mathrm{M}]$ ) that $f$ is topologically (and even Hölder) conjugate to the automorphism defined by $A$. This implies that the stable and unstable foliations of $f$ are globally defined by corresponding one-forms $\bar{\alpha}^{s}$ and $\bar{\alpha}^{u}$. In this situation we can repeat the proof of Lemma 4 in $[\mathrm{MM}]$ in a much simpler framework, since we do not have to care about smooth dependence on parameters, constructing two one-forms of class $C^{1+\delta}$, $\alpha^{s}=\phi^{s} \cdot \bar{\alpha}^{s}$ and $\alpha^{u}=\phi^{u} \cdot \bar{\alpha}^{u}$, satisfying

$$
f_{*} \alpha^{s}=\lambda^{s} \alpha^{s}, \quad f_{*} \alpha^{u}=\lambda^{u} \alpha^{u}
$$

with $0<\lambda^{s}<1<\lambda^{u}$. (If $\lambda^{s}$ or $\lambda^{u}$ are not positive, we consider $f^{2}$ instead of $f$.) 
Let us point out that as a consequence of the construction in [MM], $\alpha^{s}$ and $\alpha^{u}$ are regular along the corresponding invariant foliations, namely $\alpha^{s}$ is of class $C_{s}^{\infty}$ in the sense that its components in any local system of coordinates are $C_{s}^{\infty}$ according to Definition 2.2 in [LMM], and analogously for $\alpha^{u}$. In case $f$ is real analytic, $\alpha^{s}$ is of class $C_{s}^{\omega}$ and $\alpha^{u}$ is $C_{u}^{\omega}$. These facts are a consequence of Lemma 2.2 in [LMM] for the $C^{\infty}$ case, and estimate (2.16) and the comments that follow it in the same paper for the $C^{\omega}$ case.

The forms $\alpha^{s}, \alpha^{u}$ must be closed, as a consequence of the following facts, since $\alpha^{s} \wedge \alpha^{u}$ is a volume element with $f^{*}\left(\alpha^{s} \wedge \alpha^{u}\right)=\lambda^{s} \lambda^{u} \alpha^{s} \wedge \alpha^{u}$, taking cohomology classes we see that $\lambda^{s} \lambda^{u}=1$. Then, if $X^{s}, X^{u}$ form the dual basis to $\alpha^{s}, \alpha^{u}$, we have

$$
f_{*}\left[X^{s}, X^{u}\right]=\left[X^{s}, X^{u}\right]
$$

and by the hyperbolicity of $f_{*}$ on $C^{0}$ vector fields, we have $\left[X^{s}, X^{u}\right]=0$, hence $d \alpha^{s}=d \alpha^{u}=0$.

Notice that as a consequence of the regularity of $\alpha^{s}$ and $\alpha^{u}$, we have $X^{s} \in C^{1+\delta} \cap C_{s}^{\infty}$ and $X^{u} \in C^{1+\delta} \cap C_{u}^{\infty}$. In case $f$ is real analytic, $\infty$ can be substituted by $\omega$.

Let $\alpha_{0}^{s}, \alpha_{0}^{u}$ be the translation invariant one-forms in the same cohomology class of $\alpha^{s}$ and $\alpha^{u}$ respectively, and let $\psi^{s}, \psi^{u}$ be $C^{2+\delta}$ functions on $T^{2 n}$ verifying $\alpha^{s}=\alpha_{0}^{s}+d \psi^{s}$, and $\alpha^{u}=\alpha_{0}^{u}+\mathrm{d} \psi^{u}$. We define

$$
\Psi: T^{2} \rightarrow T^{2}, \quad x \mapsto \Psi(x)=x+\psi^{s} X_{0}^{s}+\psi^{u} X_{0}^{u},
$$

where $X_{0}^{s}, X_{0}^{u}$ form the dual basis of $\alpha_{0}^{s}, \alpha_{0}^{u}$. Then

$$
d \Psi=d x+d \psi^{s} \cdot X_{0}^{s}+d \psi^{u} \cdot X_{0}^{u}=d x+\left(\alpha^{s}-\alpha_{0}^{s}\right) X_{0}^{s}+\left(\alpha^{u}-\alpha_{0}^{u}\right) X_{0}^{u}=\alpha^{s} \cdot X_{0}^{s}+\alpha^{u} \cdot X_{0}^{u},
$$

so that

$$
\Psi^{*} \alpha_{0}^{s}=\alpha^{s}, \quad \Psi^{*} \alpha_{0}^{u}=\alpha^{u} .
$$

It is obvious then that $\Psi$ is a local diffeomorphism. Its injectivity can be proved now as follows: on one hand, $\Psi$ sends stable leaves for $f$ into stable leaves for $A$, in a monotonous way (the leaves are one-dimensional). Since the leaves are not closed this implies first that different leaves are sent to different leaves, and then that different points are sent to different points. Thus, we have proved that $\Psi$ is a diffeomorphism of class $C^{2+\delta}$. (The injectivity of $\Psi$ can also be seen as a consequence of the strong expansivity properties of the lifting of $f$ on the universal covering of $T^{2 n}$, or it can even be shown by a simple argument using degree theory).

In this situation, by (1.1) and (1.2),

$$
\left(\Psi \circ f \circ \Psi^{-1}\right)^{*} \alpha_{0}^{s}=\lambda^{s} \alpha_{0}^{s} \quad \text { and }\left(\Psi \circ f \circ \Psi^{-1}\right)^{*} \alpha_{0}^{u}=\lambda^{u} \alpha_{0}^{u} \text {, }
$$

so that $\Psi \circ f \circ \Psi^{-1}$ differs from $A$ by a translation, let us say $T_{v}$. An elementary computation shows then that

if $w=(A-I)^{-1} \cdot v$.

$$
\left(T_{w} \circ \Psi\right) \circ f \circ\left(T_{w} \circ \Psi\right)^{-1}=A
$$

Let us see now that $h=T_{w} \circ \Psi$ is of class $C^{\infty}$ if $f$ is $C^{\infty}$, and it is real analytic if $f$ is so. By (1.2),

$$
h^{\prime} \cdot X^{s}=X_{0}^{s}, \quad h^{\prime} \cdot X^{u}=X_{0}^{u}
$$


Since $X^{s}$ is of class $C_{s}^{\infty}$, by (1.3), the derivative of $h$ along the stable manifold of $f$ with respect to arc length in the same direction of $X^{s}$ is also of class $C_{s}^{\infty}$, and this implies that $h$ is of class $C_{s}^{\infty}$. In a similar way, one proves that $h$ is of class $C_{u}^{\infty}$. This in turn implies that $h^{-1}$ is of class $C_{s}^{\infty} \cap C_{u}^{\infty}$ with respect to the foliations defined by $A$. Since these foliations are of class $C^{\infty}$ (they have actually straight lines as leaves), an elementary argument using Fourier series shows that $h$ is of class $C^{\infty}$. The above argument can be used in the case of a real analytic $f$ to show that $h$ is then real analytic (see [LMM, p. 43]).

The statements concerning the area preserving case are simple to prove now: first of all, since $A$ is an invertible matrix with integer coefficients, it preserves area up to sign; since it is the matrix corresponding to $f^{*}$ on $H^{1}\left(T^{2}, \mathbb{Z}\right)$, it is area preserving. Moreover,

$$
f^{*} h^{*} \omega=h^{*} A^{*} \omega=h^{*} \omega
$$

Since $h^{*} \omega$ is equivalent to Lebesgue measure, it follows from the transitivity of $f$ that it equals a constant times $\omega$, so $h$ is area preserving.

Assume now that $f$ and $A$ are cohomologous in the sense that $f \circ A^{-1}$ is globally canonical. Since they are isotopic there is a vector field $\tilde{f} \in C^{\infty}\left(T^{2}, \mathbb{R}^{2}\right)$ such that $f=A+\tilde{f}$. Then $f \circ A^{-1}=I+\tilde{f} \circ A^{-1}$ is cohomologous to the identity, so according to [CZ, Theorem 6], $\tilde{f} A^{-1}$ (and so $\tilde{f}$ ) has vanishing average over $T^{2}$.

Since $A=f^{*}$, the conjugation equation implies that $A \circ h^{*}=h^{*} \circ A$, and since $h^{*}$ has integer coefficients this implies that $h^{*}$ is a power of $A$; substituting $h$ by itself composed with the inverse of that power, the conjugation still holds for the new $h$, and $h^{*}$ is the identity. Then $h=I+\tilde{h}$, where $\tilde{h} \in C^{\infty}\left(T^{2}, \mathbb{R}^{2}\right)$, and the conjugation equation says that

$$
\tilde{f}+\tilde{h} \circ f=A \circ \tilde{h} .
$$

Integrating both sides of this expression on $T^{2}$, and using the fact that the integral of $\tilde{f}$ vanishes, we see that

$$
\int_{T^{2}} \tilde{h}=A \int_{T^{2}} \tilde{h}
$$

and the hyperbolicity of $A$ implies that the average of $\tilde{h}$ vanishes. Again by Theorem 6 in [CZ], this means that $h$ is cohomologous to the identity. This finishes the proof of Theorem 1.

\section{The Cohomology Vector Equation, and Smooth Conjugacy of Anosov Flows}

In this section we give necessary and sufficient conditions for the regularity of solutions of the vector cohomology equations $f_{*} Y-Y=Z$, and $L_{X} Y=Z$, where $f$ is a $C^{\infty}$ Anosov diffeomorphism on the 2-dimensional torus $T^{2}$, and $X$ is a $C^{\infty}$ vector field that generates an Anosov flow. It is well known that the first equation always has a solution, and the same is true for the second equation modulo multiples of $X$. However, the solutions are in general only continuous. This fact is closely related to the fact that nearby Anosov systems are only $C^{0}$ conjugated in general (up to reparametrizations in the case of flows), according to Anosov's structural stability theorem. 
The conditions for regularity that we find are of a cohomological character, since they are relations between sums or integrals along periodic orbits of expressions involving the values of coordinates of $Z$ and their derivatives. Our results (Theorems 2 and 3) cannot be extended to higher dimensions, since the invariant foliations are not regular enough. However, there should be results analogous to Lemmas 1 and $1^{\prime}$ that hold in higher dimensions.

We apply the described results to the study of smooth conjugacy problems for one-parameter families of Anosov flows on 3-dimensional manifolds in Theorem 4.

We introduce now some notation. For a fixed $C^{\infty}$ Anosov diffeomorphism on $T^{2}$ we assume that $X^{s}, X^{u}$ are global $C^{1+\alpha} \cap C_{s}^{\infty}$ (respectively $C^{1+\alpha} \cap C_{u}^{\infty}$ ) sections of the stable and unstable bundles respectively, as at the beginning of the proof of Theorem 1. We define the functions $\lambda_{j}^{s} \in C_{s}^{\infty}(M), j \in \mathbb{Z}$, by $f_{*}^{j} X^{s}=\lambda_{j}^{s} X^{s}$. By the contractivity of $f$ on stable directions, there are constants $C>0, \lambda \in(0,1)$ such that

$$
\left|\lambda_{j}^{s}(x)\right| \leqq C \lambda^{j}, \quad \forall x \in M, \quad \forall f \in \mathbb{N}
$$

Given a continuous stable vector field $Y^{s}$ we define the continuous function $Y_{s}$ by $Y^{s}=Y_{s} \cdot X^{s}$. In case $Y^{s}$ is of class $C_{s}^{k}$, so is $Y_{s}$. We use the analogous notation for unstable vector bundles. Finally, if $Y$ is a continuous vector field, we denote by $Y^{s}$, $Y^{u}$ its components in the decomposition $T M=E^{s} \oplus E^{u}$. If $Y$ is of class $C^{1+\alpha}$, so are $Y^{s}$ and $Y^{u}$.

Theorem 2. Let $f: T^{2} \rightarrow T^{2}$ be a $C^{\infty}$ Anosov diffeomorphism, and $Y$ be a continuous vector field. If $Z=f_{*} Y-Y$ is of class $C^{\infty}$, then $Y$ is of class $C^{\infty}$ if and only if for any periodic orbit of period $N$,

$$
\sum_{j=0}^{N-1}\left(X^{s} Z_{s}\right)\left(x_{j}\right)=\frac{1}{\lambda_{N}^{s}\left(x_{0}\right)-1} \cdot \sum_{j=0}^{N-1} \lambda_{k}^{s}\left(x_{j}\right) \cdot Z_{s}\left(x_{j-k}\right) \cdot\left(X^{s} \lambda_{1}^{s}\right)\left(x_{j+1}\right)
$$

and similarly for the unstable component $Z_{u}$ of $Z$.

The proof of Theorem 2 will be postponed until we prove an analogous result for flows (Theorem 3). Before stating the corresponding result for flows we introduce the appropriate notation.

By the stable foliation of an Anosov flow $\phi_{t}$ in a 3-dimensional manifold $M$ we mean the foliation by (one-dimensional) contracting leaves. The corresponding foliation by (two-dimensional) stable manifolds of orbits will be called the centerstable foliation. A map or function will be said to be of class $C_{s}^{k}$, just as in [LMM] where these spaces were introduced, if its restriction to each center-stable manifold is of class $C^{k}$ and the $k$-jets of these restrictions depend continuously on the point in $M$. Finally, it will be said to be of class $C_{X}^{k}$ when it satisfies the corresponding properties on orbits of the vector field $X$ that generates the flow.

We assume that the vector fields $X^{s}, X^{u}$ are $C^{\alpha} \cap C_{s}^{\infty}$ (respectively $C^{\alpha} \cap C_{u}^{\infty}$ ) sections of the stable and unstable bundles respectively. Notice that as a consequence of the results in [P] we cannot expect $X^{s, u}$ to be of class $C^{1}$. We define the function $l^{s} \in C_{s}(M)$ by $L_{X} X^{s}=l^{s} X^{s}$, and we set $L^{s}\left(r, x_{0}\right)=\int_{0}^{r}\left(l^{s} \circ \phi_{-v}\right)\left(x_{0}\right) d v$, 
$F^{s}\left(r, x_{0}\right)=\exp \left[-L^{s}\left(r, x_{0}\right)\right]$. Since the flow is Anosov, there exist $K \in \mathbb{R}, C>0$ such that

$$
L^{s}\left(r, x_{0}\right) \geqq K+C \cdot r
$$

Just as in the case of diffeomorphisms, given a continuous stable vector field $Y^{s}$ we define the continuous function $Y_{s}$ by $Y^{s}=Y_{s} \cdot X^{s}$. If $Y^{s}$ is of class $C_{s}^{k}$, so is $Y_{s}$. We use the analogous notation for unstable vector fields. Finally, if $Y$ is a continuous vector field, we denote by $Y_{0}, Y^{s}, Y^{u}$ its components in the decomposition $T M=\mathbb{R} X \oplus E^{s} \oplus E^{u}$. If $Y$ is of class $C^{1}, Y^{s}$ is of class $C_{s}^{1}$ and $Y^{u}$ is of class $C_{u}^{1}$, since the center-stable and center-unstable foliations are of class $C^{1+\alpha}$ (this wellknown fact is proved for Anosov diffeomorphisms in [HP, Theorem 6.3]. The proof can be trivially adapted to the case of Anosov flows).

Theorem 3. Let $X$ be a $C^{\infty}$ vector field on a 3-dimensional compact manifold $M$; assume $X$ generates a transitive Anosov flow. Let $Y$ be a vector field of class $C_{X}^{1}$. If $L_{X} Y=Z$ is of class $C^{\infty}$, then $Y$ is of class $C^{\infty}$ if and only if for any periodic orbit $\gamma$ of period $T$,

$$
\int_{0}^{T}\left(X^{s} Z_{s}\right)(\gamma(t)) d t=\frac{1}{F^{s}(T, \gamma(0))-1} \int_{0}^{T} \int_{0}^{T}\left(X^{s} l^{s}\right)(\gamma(t)) \cdot F^{s}(r, \gamma(t)) \cdot Z_{s}(\gamma(t-r)) d r d t
$$

and similarly for the unstable component of $Z$.

Remark. For a fixed Anosov flow and a fixed vector field $Z$, all the functions appearing in (2.4), namely $Z_{s}, F^{s}, l^{s}$ depend on the choice of a non-vanishing stable vector field $X^{s}$ of class $C_{s}^{\infty}$. However, if (2.4) holds for one choice of $X^{s}$, then it also holds for any other choice. This is a consequence of the proof of Theorem 3 that will be given below, since we shall prove that if (2.4) holds for a specific choice of $X^{s}$, then $Y$ is smooth, and we shall also prove that $Y^{s}$ being smooth implies that (2.4) holds for any choice of $X^{s}$.

Proof of Theorem 3. The proof is based on the following two lemmas:

Lemma 1. Let $Y^{s}$ be a stable vector field of class $C_{X}^{1}$. If $L_{X} Y^{s}=Z^{s}$ is of class $C_{s}^{k}, k \geqq 1$, then $Y^{s}$ is of class $C_{s}^{k}$ if and only if for any periodic orbit $\gamma$ of period $T,(2.4)$ holds. The corresponding statement for unstable vector fields is also true.

For the second lemma we need some extra notation. Given a $\mathrm{C}^{\infty}$ onedimensional vector subbundle $\hat{E}^{u}$ of $T M$, transversal to the center-stable subbundle $E^{\bar{s}}$, and given any continuous vector field $Y$, we define $\hat{Y}^{\bar{s}} \in E^{\bar{s}}, \hat{Y}^{u} \in E^{u}$ by $Y=\hat{Y}^{\bar{s}}+\hat{Y}^{u}$. If $Y$ is of class $C_{s}^{\infty}$, then $\hat{Y}^{\bar{s}}, \hat{Y}^{u}$ are also of class $C_{s}^{\infty}$.

Lemma 2. Let $Y$ be a vector field of class $C_{X}^{1}$. If $L_{X} Y=Z$ is of class $C^{\infty}$, then $\hat{Y}^{u}$ is of class $C_{s}^{\infty}$.

We show now how Theorem 3 is a consequence of these two lemmas, and than their proof will be given.

Assume that $Y$ is a $C^{\infty}$ vector field. By the remarks preceding Lemma 2, $Y^{s}$ is of class $C_{s}^{1}$; morevoer, $L_{X} Y^{s}=Z^{s}$ is also of class $C_{s}^{1}$, so we can apply Lemma 1 and we see that (2.4) is satisfied. The corresponding formula for the unstable component is proved in the same way. 
Assume now that $L_{X} Y=Z$ is a $C^{\infty}$ vector field and it satisfies (2.4). Let $\hat{E}^{u}$ be a smooth subbundle of $T M$ as in Lemma 2. According to this lemma, $\hat{Y}^{u}$ is of class $C_{s}^{\infty}$. Assume

$$
\hat{Y}^{\bar{s}}=\hat{Y}^{s}+\mu X
$$

where $\hat{Y}^{s} \in E^{s}$. Then $L_{X} \hat{Y}^{s}$ is the stable component of the $C_{s}^{\infty}$ center-stable vector field $Z-L_{X} \hat{Y}^{u}$. Since the splitting $E^{\bar{s}}=E^{s} \oplus \mathbb{R} X$ is of class $C_{s}^{\infty}$, we see that $L_{X} \hat{Y}^{s}$ is a stable vector field of class $C_{s}^{\infty}$. Since $Y=\hat{Y}^{s}+\mu X+\hat{Y}^{u}$, we see that

$$
Z=L_{X} \hat{Y}^{s}+(X \mu) X+L_{X} \hat{Y}^{u} .
$$

Taking the stable components in both sides we have

$$
L_{X} \hat{Y}^{s}=Z^{s}-L_{X}\left(\hat{Y}^{u}\right)^{s} .
$$

Since both $\left(\hat{Y}^{u}\right)^{s}$ and $L_{X}\left(\hat{Y}^{u}\right)^{s}$ are of class $C_{s}^{1}$, by Lemma $1,\left(L_{X} \hat{Y}^{u}\right)_{s}$ satisfies the same condition $Z_{s}$ does in (2.4); by our assumption on $Z$, and the linearity of (2.4) on $Z_{s}$, $\left(Z-L_{X} \int_{0}^{n} \hat{Y}^{u}\right)_{s}$ also satisfies that condition, so again by Lemma $1, \hat{Y}^{s}$ is a $C_{s}^{\infty}$ vector field.

On the other hand, taking components along $X$ in (2.6) we see that $X \mu$ is the component along $X$ of $Z-L_{X} \hat{Y}^{u}$, so it is of class $C_{s}^{\infty}$. By Lemma 2.2 in [LMM], $\mu$ itself is of class $C_{s}^{\infty}$. This proves that $Y=\hat{Y}^{s}+\mu X+\hat{Y}^{u}$ is of class $C_{s}^{\infty}$.

A similar argument shows that if the unstable component of $Z$ satisfies the condition that corresponds to (2.4), then $Y$ is of class $C_{u}^{\infty}$. By Lemma 2.3 in [LMM] it follows that $Y$ is of class $C^{\infty}$. This finishes the proof of Theorem 3 .

Proof of Lemma 1. The fact that $Z^{s}$ and $Y^{s}$ being of class $C_{s}^{1}$ implies (2.4) has a local character for each periodic orbit $\gamma$; it is actually a simple property of attracting periodic orbits of flows in two dimensions, since only the local stable manifold of $\gamma$ is involved. The proof goes as follows:

By the definitions of $l^{s}, Y_{s}$ and $Z_{s}$, the equality $L_{X} Y^{s}=Z^{s}$ is equivalent to

$$
X Y_{s}+l^{s} Y_{s}=Z_{s}
$$

By (2.3), the previous expression implies that

$$
Y^{s}\left(x_{0}\right)=\int_{0}^{\infty} F^{s}\left(r, x_{0}\right) \cdot\left(Z_{s} \circ \phi_{-r}\right)\left(x_{0}\right) d r .
$$

Since $F^{s}(a+b, x)=F^{s}\left(a, \phi_{-b}(x)\right) \cdot F^{s}(b, x)$, we see that on $\gamma$,

$$
\begin{aligned}
Y^{s}\left(x_{0}\right) & =\sum_{k=0}^{\infty} \int_{0}^{T} F^{s}\left(k T+r, x_{0}\right) \cdot z_{s}(\gamma(-r)) d r d t \\
& =\frac{1}{F^{s}\left(T, x_{0}\right)-1} \int_{0}^{T} F^{s}\left(r, x_{0}\right) \cdot z_{s}(\gamma(-r)) d r .
\end{aligned}
$$

If we differentiate along $X^{s}$ in (2.7), taking into account the definition of $l^{s}$ and (2.9), 
we see that

$$
\begin{aligned}
{\left[X\left(X^{s} Y_{s}\right)\right](\gamma(t))=} & \left(X^{s} Z_{s}\right)(\gamma(t))-\left(X^{s} l^{s}\right)(\gamma(t)) \cdot Y_{s}(\gamma(t)) \\
= & \left(X^{s} Z_{s}\right)(\gamma(t))-\left(X^{s} l^{s}\right)(\gamma(t)) \\
& \cdot \frac{1}{F^{s}(t, \gamma(t))-1} \int_{0}^{T} F^{s}(r, \gamma(t)) \cdot z_{s}(\gamma(t-r)) d r .
\end{aligned}
$$

Integrating the above expression between $t=0$ and $T$, the term in the left cancels out and we get (2.4).

Let us assume now that $Z_{s}$ satisfies (2.4). Consider the sequence of functions

$$
Y_{s}^{n}\left(x_{0}\right)=\int_{0}^{n} F^{s}\left(r, x_{0}\right) \cdot\left(Z_{s} \circ \phi_{-r}\right)\left(x_{0}\right) d r
$$

Then by (2.8), $Y_{s}=\lim _{n \rightarrow \infty} Y_{s}^{n}$ uniformly on $M$, each $Y_{s}^{n}$ is of class $C_{s}^{\infty}$ on $M$, and its derivative along $X^{s}$ can be computed directly:

$$
X^{s} Y_{s}^{n}\left(x_{0}\right)=\int_{0}^{n}\left[\left(X^{s} Z_{n}\right) \circ \phi_{-r}\right]\left(x_{0}\right) d r+\int_{0}^{n}\left(X^{s} F^{s}\right)\left(r, x_{0}\right) \cdot\left(Z_{s} \circ \phi_{-r}\right)\left(x_{0}\right) d r .
$$

Adding and subtracting the integral of $\left[\left(X^{s} l^{s}\right) \cdot Y_{s}\right] \circ \phi_{-r}$, and using (2.8),

$$
\begin{aligned}
X^{s} Y_{s}^{n}\left(x_{0}\right)= & \int_{0}^{n}\left\{\left[X^{s} Z_{n}-\left(X^{s} l^{s}\right) \cdot Y_{s}\right] \circ \phi_{-r}\right\}\left(x_{0}\right) d r \\
& +\int_{0}^{n}\left[\left(X^{s} l^{s}\right) \circ \phi_{-r}\right]\left(x_{0}\right) \int_{0}^{\infty} \exp \left[-\int_{0}^{t}\left(l^{s} \circ \phi_{-r-u}\right)\left(x_{0}\right) d u\right] \\
& \cdot\left(Z_{s} \circ \phi_{-r-t}\right)\left(x_{0}\right) d t d r \\
& -\int_{0}^{n} F^{s}\left(r, x_{0}\right) \int_{0}^{r} \exp \left[L^{s}\left(s, x_{0}\right)\right] \cdot\left[\left(X^{s} l^{s}\right) \circ \phi_{-s}\right]\left(x_{0}\right) d s \cdot\left(Z_{s} \circ \phi_{-r}\right)\left(x_{0}\right) d r .
\end{aligned}
$$

In the second integral we make the change of variables $\tilde{r}=r, \tilde{t}=r+t$, and change the order of integration. In this way we get the sum of two integrals, one over $0 \leqq \tilde{r} \leqq \tilde{t}$ $\leqq n$, and the second over $0 \leqq \tilde{r} \leqq n \leqq \tilde{t}$. The first of these two integrals cancels out with the last term in (2.10), so we have, using (2.8) again,

$$
\begin{aligned}
X^{s} Y_{s}^{n}\left(x_{0}\right)= & \int_{0}^{n}\left\{\left[X^{s} Z_{s}-\left(X^{s} l^{s}\right) \cdot Y_{s}\right] \circ \phi_{-r}\right\}\left(x_{0}\right) d r \\
& +\int_{n}^{\infty} \int_{0}^{n} \exp \left[-\int_{r}^{t}\left(l^{s} \circ \phi_{-v}\right)\left(x_{0}\right) d v\right] \cdot\left[\left(X^{s} l^{s}\right) \circ \phi_{-r}\right]\left(x_{0}\right) \cdot\left(Z_{s} \circ \phi_{-t}\right)\left(x_{0}\right) d r d t \\
= & \int_{0}^{n}\left\{\left[X^{s} Z_{s}-\left(X^{s} l^{s}\right) \cdot Y_{s}\right] \circ \phi_{-r}\right\}\left(x_{0}\right) d r \\
& +\left(Y_{s} \circ \phi_{-n}\right)\left(x_{0}\right) \cdot \int_{0}^{n} F^{s}\left(n-r, \phi_{-r}\left(x_{0}\right)\right) \cdot\left[\left(X^{s} l^{s}\right) \circ \phi_{-r}\right] d r .
\end{aligned}
$$


Since $X^{s}$ is of class $C^{1+\alpha}, l^{s}$ is of class $C^{\alpha}$, so $X^{s} Z_{s}-\left(X^{s} l^{s}\right) \cdot Y_{s}$ is of class $C^{\alpha}$. By (2.4) and Livsic's theorem (see [Li]), there exists a Hölder function $H$ on $M$ of class $C_{X}^{1}$ such that

$$
X H=X^{s} Z_{s}-\left(X^{s} l^{s}\right) \cdot Y_{s}
$$

Notice that it is at this point of the proof where we need the transitivity of $X$. Substituting this in the above expression we see that

$$
\begin{aligned}
X^{s} Y_{s}^{n}\left(x_{0}\right)= & \left(H \circ \phi_{-n}\right)\left(x_{0}\right)-H\left(x_{0}\right) \\
& +\left(Y^{s} \circ \phi_{-n}\right)\left(x_{0}\right) \int_{0}^{n} F^{s}\left(n-r, \phi_{-r}\left(x_{0}\right)\right) \cdot\left[\left(X^{s} l^{s}\right) \circ \phi_{-r}\right] d r .
\end{aligned}
$$

The first two terms are bounded in absolute value by a constant independent of $n$, and the same happens to $\left(Y^{s} \circ \phi_{-n}\right)\left(x_{0}\right)$. Finally, by $(2.3)$,

$$
\left.\mid \int_{0}^{n} F^{s}\left(n-r, \phi_{-r} x_{0}\right)\right) \cdot\left[\left(X^{s} l^{s}\right) \circ \phi_{-r}\right]\left(x_{0}\right) d r \mid \leqq \bar{C} \int_{0}^{n} \exp [-K-C(n-r)] d r
$$

is also bounded by a constant independent of $n$.

This implies that $Y_{s}=\lim _{n \rightarrow \infty} Y_{s}^{n}$ is uniformly Lipschitz along center-stable manifolds. Then it is differentiable along center-stable manifolds almost everwhere, and by (2.7), $X Y_{s}$ is also differentiable almost everywhere along center-stable manifolds. Since $X, X^{s}$ are $C^{\infty}$ vector fields along these manifolds, $X X^{s} Y_{s}$ exists in the sense of distributions on any center-stable manifold, it is a bounded function, and the same argument of the beginning of the proof of this lemma shows that

$$
X X^{s} Y_{s}=X^{s} Z_{s}-\left(X^{s} l^{s}\right) Y_{s} .
$$

By Lyvsic's theorem, $X^{s} Y_{s}$ is a Hölder function, so $Y_{s}$ is of class $C_{s}^{1}$. But this implies that the right-hand side in the above formula is of class $C_{s}^{1}$; the proof of Lemma 2.2 in [LMM] gives then that $X^{s} Y_{s}$ is of class $C_{s}^{1}$, hence $Y_{s}$ is of class $C_{s}^{2}$. The bootstrap argument can be continued to prove $Y_{s}$ is of class $C_{s}^{\infty}$. This finishes the proof of Lemma 1.

Proof of Lemma 2. Given $\tau \in \mathbb{R}$, consider the vector field $X_{\tau}=X+\tau Z$. By Anosov's persistence theorem, $X_{\tau}$ generates an Anosov flow if $\tau$ is close to 0 .

By the smooth dependence on parameters in Anosov's structural stability theorem (see Theorem A.1 in [LMM] for a detailed proof), there exists a $C^{\infty}$ family of homeomorphisms $h_{\tau}$, and a $C^{\infty}$ family of continuous functions on $M \xi_{\tau}$, such that $h_{\tau}$ conjugates the flows of $X_{0}$ and $\xi_{\tau} \cdot X_{\tau}$. Moreover, $h_{\tau}$ transforms the center-stable manifold of $x_{0} \in M$ with respect to $X_{0}$ into the center-stable manifold of $h_{\tau}\left(x_{0}\right)$ with respect to $X_{\tau}$.

Lemma 2 will be a consequence of this and the smooth dependence of the centerstable spaces on parameters, as stated in the follwing lemma:

Lemma 3. Let $X_{\tau}$ be a $C^{\infty}$ family of $C^{\infty}$ vector fields that generate Anosov flows on the compact 3-dimensional manifold $M$. Then the map that sends each $(x, \tau) \in M \times I$ to the center-stable subspace $E_{x, \tau}^{\bar{s}}$ of $X_{\tau}$ at $x$ is of class $C^{1}$ as a section of the Grassmanian bundle over $M$. Moreover, its derivative with respect to tdefines a $C_{s}^{\infty}$ map. If Tis a $C^{\infty}$ vector field, the derivative of $E_{x, \tau}^{\bar{s}}$ along $T$ is also a $C_{s}^{\infty}$ map. 
Taking Lemma 3 for granted, we shall finish the proof of Lemma 2. We restrict ourselves to a small neighbourhood of an arbitrary point $\mathrm{x}_{0} \in M$, where we assume $\hat{E}^{u}$ is generated by $\partial / \partial x_{1}$ for some system of coordinates $\left(x_{1}, x_{2}, x_{3}\right)$. We can also assume that the local center-stable manifold of $x_{0}$ with respect to $X_{0}$ is given by $x_{1}=0, X_{0}=\partial / \partial x_{2}$, and $E_{x, \tau}^{\bar{s}}$ is generated by $\frac{\partial}{\partial x_{j}}+m_{\tau}^{j}(x) \cdot \frac{\partial}{\partial x_{1}}$, for $j=2,3$, where $m_{\tau}^{j}, \partial m_{\tau}^{j} / \partial x_{1}$ and $d m_{\tau}^{j} / d \tau$ are functions of class $C_{s}^{\infty}$ for $\tau=0$, and they are of class $C^{1}$ jointly on $x$ and $\tau$.

Then the center-stable map of $X_{\tau}$ through $h_{\tau}\left(x_{0}\right)$ is given by a function $x_{1}=\eta_{\tau}\left(x_{2}, x_{3}\right)$ satisfying

and

$$
\frac{\partial \eta_{\tau}}{\partial x_{j}}=m_{\tau}^{j}\left(\eta_{\tau}, x_{2}, x_{3}\right), \quad j=2,3
$$

$$
\eta_{\tau}\left(x_{2}^{\tau}, x_{3}^{\tau}\right)=x_{1}^{\tau}
$$

where $\left(x_{1}^{\tau}, x_{2}^{\tau}, x_{3}^{\tau}\right)$ are the coordinates of $h_{\tau}\left(0, x_{2}, x_{3}\right)$.

By the smooth dependence of solutions of ordinary differential equations on initial conditions and parameters, $\eta_{\tau}$ can be differentiated with respect to $\tau$, and we can also differentiate the last two expressions with respect to $\tau$. If we denote derivatives with respect to $\tau$ by ', and taking into account that $\eta_{0} \equiv 0$, we get the following making $\tau=0$ :

and

$$
\frac{\partial \eta_{0}}{\partial x_{j}}=\dot{m}_{0}^{j}\left(0, x_{2}, x_{3}\right)+\frac{\partial m_{0}^{j}}{\partial x_{1}}\left(0, x_{2}, x_{3}\right) \cdot \dot{\eta}_{0}
$$

$$
\dot{\eta}_{0}\left(x_{2}, x_{3}\right)=\dot{x}_{0}^{1}
$$

By (2.12), the component along $\hat{E}^{u}$ of $\dot{h}$ at points of the center-stable manifold of $x_{0}$ is just $\dot{\eta}_{0}$. By (2.11), this map is of class $C^{\infty}$ along that center-stable manifold. Moreover, since $\dot{m}_{0}^{j}, \partial m_{0}^{j} / \partial x_{1}$ depend continuously on the choice we made of the parametrization of the center-stable manifold, the component along $\hat{E}^{u}$ of $\dot{h}$ is of class $C_{s}^{\infty}$ on $M$. We claim that this component is also the component of $Y$ along $\hat{E}^{u}$, and this will finish the proof of Lemma 2. To see this we differentiate

$$
h_{\tau}^{*} X_{\tau}=\xi_{\tau} \cdot X_{0}
$$

with respect to $\tau$ at $\tau=0$, and we have

$$
L_{X} \dot{h}_{0}=Z+\dot{\xi}_{0} X
$$

Since $L_{X} Y=Z$, we see that $Y=\dot{h}_{0}-\eta X$, where $X \eta=\dot{\xi}_{0}$. Taking components along $\hat{E}^{u}$ on the center-stable manifold of $x_{0}$ our claim is proved, and this finishes the proof of Lemma 2.

Proof of Lemma 3. The fact that $E_{x, \tau}^{\bar{s}}$ has $C^{1}$ dependence on $x$ and $\tau$ is a direct consequence of the $C^{r}$ section theorem (Theorem 3.5 in [HPS]). The corresponding result for Anosov diffeomorphisms on $T^{2}$ is proved in the first part of Lemma 4 in $[\mathrm{MM}]$, and the same proof can be extended immediately to our case. 
Consider two vector subbundles of $T M, \widetilde{E}^{\bar{s}}, \widetilde{E}^{u}$, and assume they are uniformly close enough to $E_{\tau}^{\bar{s}}, E_{\tau}^{u}$ for $|\tau|<\varepsilon_{0}$. Then the center stable space $E_{x, \tau}^{\bar{s}}$ is the graph of a linear map $\sigma_{\tau}(x) \in L\left(\widetilde{E}_{x}^{\bar{s}}, \widetilde{E}_{x}^{u}\right)$, uniformly close to 0 . The invariance of $E_{x, \tau}^{\bar{s}}$ under the flow implies that

$$
\sigma_{\tau}\left(f_{\tau}(x)\right) \cdot\left(f_{\tau}^{11}(x)+f_{\tau}^{12}(x) \cdot \sigma_{\tau}(x)\right)=f_{\tau}^{21}(x)+f_{\tau}^{22}(x) \cdot \sigma_{\tau}(x),
$$

where $f_{\tau}$ is the time- $(-1)$ flow of $X_{\tau}$, and the tangent map $f_{\tau}^{\prime}(x): T_{x} M \rightarrow T_{f_{\tau}(x)} M$ defines corresponding linear maps $f_{\tau}^{i j}(x)$ under the splittings $T_{x} M=\widetilde{E}_{x}^{\bar{s}} \oplus \widetilde{E}_{x}^{u}$ and $T_{f_{\tau}(x)} M=\widetilde{E}_{f_{\tau}(x)}^{\bar{s}} \oplus \widetilde{E}_{f_{\tau}(x)}^{u}$.

We want to take derivatives with respect to $\tau$ in (2.13), but the dependence on $\tau$ of the fibers where each linear map is defined would make the resulting formula unuseful if we did it directly. Instead of that, we shall first compose with suitable fibered maps that send the fiber over $f_{\tau}(x)$ to the fiber over $f(x)$.

Given two points in $M$ close enough, we consider the linear map $L_{x, y}: T_{x} M \rightarrow T_{y} M$ given by parallel translation along the shortest geodesic that joins $x$ and $y$ with respect to some fixed riemannian metric, and we set

$$
\begin{gathered}
\tilde{\sigma}_{\tau}\left(f_{0}(x)\right)=L_{f_{0}(x), f_{\tau}(x)} \cdot \sigma_{\tau}\left(f_{\tau}(x)\right) \cdot L_{f_{\tau}(x), f_{0}(x)} \in L\left(T_{f_{0}(x)} M, T_{f_{0}(x)} M\right), \\
\tilde{f}_{\tau}^{i j}(x)=L_{f_{0}(x), f_{\tau}(x)} \cdot f_{\tau}^{i j}(x) \in L\left(T_{x} M, T_{f_{0}(x)} M\right) .
\end{gathered}
$$

Applying $L_{f_{0}(x), f_{\tau}(x)}$ to both sides of (2.13) we get

$$
\tilde{\sigma}_{\tau}\left(f_{0}(x)\right) \cdot\left(\tilde{f}_{\tau}^{11}(x)+\tilde{f}_{\tau}^{12}(x) \cdot \sigma_{\tau}(x)\right)=\tilde{f}_{\tau}^{21}(x)+\tilde{f}_{\tau}^{22}(x) \cdot \sigma_{\tau}(x) .
$$

If we differentiate (2.14) with respect to $\tau$ we have

$$
\begin{aligned}
& \dot{\tilde{\sigma}}_{\tau}\left(f_{0}(x)\right) \cdot\left(\tilde{f}_{\tau}^{11}(x)+\tilde{f}_{\tau}^{12}(x) \cdot \sigma_{\tau}(x)\right)+\tilde{\sigma}_{\tau}(f(x)) \cdot\left(\dot{\tilde{f}}_{\tau}^{11}(x)\right. \\
& \left.\quad+\dot{\tilde{f}}_{\tau}^{12}(x) \cdot \sigma_{\tau}(x)+\tilde{f}_{\tau}^{12}(x) \cdot \sigma_{\tau}(x)\right)=\dot{f}_{\tau}^{21}(x)+\widetilde{f}_{\tau}^{22}(x) \cdot \sigma_{\tau}(x)+\tilde{f}_{\tau}^{22}(x) \cdot \dot{\sigma}_{\tau}(x) .
\end{aligned}
$$

If we denote for a given section $\sigma$ of $L(T M, T M)$,

$$
F_{\tau} \sigma\left(f_{0}(x)\right)=L_{f_{0}(x), f_{\tau}(x)} \cdot \sigma\left(f_{\tau}(x)\right) \cdot L_{f_{\tau}(x), f_{0}(x)} \in L\left(T_{f_{0}(x)} M, T_{f_{0}(x)} M\right)
$$

then we see that $\tilde{\sigma}_{\tau}=F_{\tau} \sigma_{\tau}$, so that since $F_{\tau}$ is linear, $\dot{\sigma_{\tau}}=\dot{F}_{\tau} \sigma_{\tau}+F_{\tau} \dot{\sigma}_{\tau}$. Using this and applying $L_{f_{\tau}(x), f_{0}(x)}$ to both sides of (2.15), we have

$$
\begin{aligned}
{\left[\dot{\sigma}_{\tau}\left(f_{\tau}(x)\right)+\right.} & \left.L_{f_{\tau}(x), f_{0}(x)}\left(\dot{F}_{\tau} \sigma_{\tau}\right) L_{f_{0}(x), f_{\tau}(x)}\right] \cdot\left[f_{\tau}^{11}(x)+f_{\tau}^{12}(x) \cdot \sigma_{\tau}(x)\right] \\
& +\sigma_{\tau}\left(f_{\tau}(x)\right) \cdot L_{f_{0}(x), f_{\tau}(x)}\left[\dot{\vec{f}}_{\tau}^{11}(x)+\dot{\tilde{f}}_{\tau}^{12}(x) \cdot \sigma_{\tau}(x)\right]+\sigma_{\tau}\left(f_{\tau}(x)\right) \\
& \cdot f_{\tau}^{12}(x) \cdot \dot{\sigma}_{\tau}(x)=L_{f_{\tau}(x), f_{0}(x)}\left[\dot{\tilde{f}}_{\tau}^{21}(x)+\dot{\tilde{f}}_{\tau}^{22}(x) \cdot \sigma_{\tau}(x)\right]+f_{\tau}^{22}(x) \cdot \dot{\sigma}_{\tau}(x)
\end{aligned}
$$

or, equivalently,

$$
\begin{aligned}
\dot{\sigma}_{\tau}\left(f_{\tau}(x)\right)= & {\left[f_{\tau}^{22}(x)-\sigma_{\tau}\left(f_{\tau}(x)\right) \cdot f_{\tau}^{12}(x)\right] \dot{\sigma}_{\tau}(x)\left[f_{\tau}^{11}(x)+f_{\tau}^{12}(x) \cdot \sigma_{\tau}(x)\right]^{-1} } \\
& +\left\{L_{f_{\tau}(x), f_{0}(x)}\left[\tilde{f}_{\tau}^{21}(x)+\dot{\tilde{f}}_{\tau}^{22}(x) \cdot \sigma_{\tau}(x)\right]-\sigma_{\tau}(f(x))\right. \\
& \left.\cdot L_{f_{0}(x), f_{\tau}(x)} \cdot\left[\tilde{f}_{\tau}^{11}(x)+\tilde{f}_{\tau}^{12}(x) \cdot \sigma_{\tau}(x)\right]\right\} \cdot\left[f_{\tau}^{11}(x)+f_{\tau}^{12}(x) \cdot \sigma_{\tau}(x)\right]^{-1} \\
& -L_{f_{\tau}(x), f_{0}(x)} \cdot\left(\dot{F}_{\tau} \sigma_{\tau}\right) \cdot L_{f_{0}(x), f_{\tau}(x)} .
\end{aligned}
$$


The right-hand side in this expression defines a Lipschitz fibered map on the linear bundle $L(T M, T M)$ over $f_{\tau}$. This map involves $\sigma_{\tau}$, which is not $C^{\infty}$, but only $C_{s}^{\infty}$, so it is of class $C_{\mathscr{F}_{s}}^{\infty}$, where $\mathscr{F}_{s}$ is the foliation of $L(T M, T M)$ whose leaves are the unions of the fibers of all points in a center-stable manifold of $f_{\tau}$. Moreover, it is a fiber-contracting map, since only the first term contributes to the Lipschitz constant along fibers, and both $f_{\tau}^{22}(x)$ and $\left(f_{\tau}^{11}(x)\right)^{-1}$ are uniformly contracting, and the whole term involves small perturbations of them if $\widetilde{E}^{\bar{s}}, \widetilde{E}^{u}$ are close enough to $E^{\bar{s}}, E^{u}$ respectively. Then Lemma 3 will be a consequence of the following variation of the $C^{r}$ section theorem:

Lemma 4. Let $f: M \rightarrow M$ be a diffeomorphism, $\pi: E \rightarrow M$ a fiber bundle, and $F: E \rightarrow E$ a Lipschitz fiber contraction over $f$. Assume $\mathscr{F}$ is $C^{0}$ foliation by $C^{\infty}$ leaves, invariant with respect to $f$, and uniformly contracting. If $F$ is of class $C_{\pi^{*} \mathscr{F}}^{\infty}$ then the invariant section is of class $C_{\mathscr{F}}^{\infty}$.

The proof is identical to the usual proofs of the $C^{r}$ case in [HP, Sh, HPS], and will be omitted.

Using Lemma 4 we see that $\dot{\sigma}_{\tau}$, and hence the derivative of $E_{x, \tau}^{\bar{s}}$ with respect to $\tau$, is $C^{\infty}$ along stable (strictly contracting) directions. We shall prove now that is also $C^{\infty}$ along the flow of $X$ (that is, it is in the class $C_{X}^{\infty}$ ). These two facts imply that it is of class $C_{s}^{\infty}$.

The $C_{X}^{\infty}$ regularity can be proved as follows: the subbundles $E_{\tau}^{\bar{s}}$ of $T_{x} M$ are the kernels of suitable $C^{1} \cap C_{s}^{\infty}$ one-forms $\alpha_{\tau}^{s}$ satisfying

$$
L_{X_{\tau}} \alpha_{\tau}^{s}=\lambda_{\tau}^{s} \alpha_{\tau}^{s}
$$

for some $C^{1} \cap C_{s}^{\infty}$ functions $\lambda_{\tau}^{s}$ which show also $C^{1}$ dependence with respect to $\tau$.

Differentiating the above expression with respect to $\tau$ we see that

$$
L_{X_{\tau}} \dot{\alpha}_{\tau}+L_{\dot{X}_{\tau}} \alpha_{\tau}=\dot{\lambda}_{\tau}^{s} \alpha_{\tau}+\lambda_{\tau}^{s} \dot{\alpha}_{\tau}
$$

which can be seen as an ordinary differential equation for $\dot{\alpha}_{\tau}$ along the integral curves of $X_{\tau}$ with $C_{X}^{\infty}$ coefficients. It follows that $\dot{\alpha}_{\tau}$, and hence $\dot{E}^{\bar{s}}$, are of class $C_{X}^{\infty}$.

Finally, if $T$ is a $C^{\infty}$ vector field, we have to prove that the derivative of $E_{x, \tau}^{\bar{s}}$ along $T$ is a $C_{s}^{\infty}$ map. This can be reduced to the previous case as follows. First, we can just look at the case $\tau=0$. If $\phi_{t}, t \in \mathbb{R}$, is the flow of $T$, we consider $\bar{X}_{t}=\phi_{t}^{*} X_{0}$. Let $\bar{E}_{x, t}^{\bar{s}}$ be its corresponding center-stable spaces. Then $E_{\phi_{t}(x), 0}^{\bar{s}}=\phi_{t}^{\prime} \bar{E}_{x, t}^{\bar{s}}$, and by the previous case it can be differentiated explicitly with respect to $t$. This finishes the proof of Lemma 3.

We give now a proof of Theorem 2. It follows closely that of Theorem 3 . Only those parts of the proof which are not direct translations of the case of flows will be worked out in detail.

Just like Theorem 3, the proof of Theorem 2 is based on two lemmas, as follows:

Lemma $1^{\prime}$. Let $Y^{s}$ be a continuous stable vector field. If $Z^{s}=f_{*} Y^{s}-Y^{s}$ is of class $C_{s}^{k}$, $k \geqq 1$, then $Y^{s}$ is of class $C_{s}^{k}$ if and only if for any periodic orbit of period $N,(2.2)$ holds. The corresponding statement for unstable vector fields is also true.

Assume now that $\hat{E}^{u}$ is a $C^{\infty}$ one-dimensional vector subbundle of $T M$, transversal to the stable subbundle $E^{s}$. Given any continuous vector field $Y$, define 
$\hat{Y}^{s} \in E^{s}, \hat{Y}^{u} \in \hat{E}^{u}$ by imposing $Y=\hat{Y}^{s}+\hat{Y}^{u}$. If $Y$ is of class $C_{s}^{\infty}$, then $\hat{Y}^{s}, \hat{Y}^{u}$ are also of class $C_{s}^{\infty}$.

Lemma $2^{\prime}$. If $Y$ is a continuous vector field and $Z=f_{*} Y-Y$ is of class $C^{\infty}$, then $\hat{Y}^{u}$ is of class $C_{s}^{\infty}$.

The proof of Theorem 2 from the above two lemmas is identical to the case of flows and it is left to the reader. On the other hand, Lemma $2^{\prime}$ is a consequence of Lemma 2: given $Y$ and $\hat{E}^{u}$ on $M$, if the corresponding suspended flow is defined on $\tilde{M}=M \times \mathbb{R} / \sim$, we can construct a corresponding vector field $\tilde{Y}$ on $\tilde{M}$ by

$$
\tilde{Y}(x, t)=\sum_{j=-\infty}^{\infty} \chi(t-j) \cdot f_{*}^{j} Y
$$

where $\chi \in C_{0}^{\infty}(-1,1)$ satisfies $\sum_{j=-\infty}^{\infty} \chi(t-j)=1$ (notice that the above infinite sums have at most two non-vanishing terms), and then

$$
L_{\partial / \partial t} \tilde{Y}=\sum_{j=-\infty}^{\infty} \chi^{\prime}(t-j) \cdot f_{*}^{j} Y=\frac{1}{2} \sum_{j=-\infty}^{\infty} \chi^{\prime}(t-j) \cdot\left(f_{*}^{j} Y-f_{*}^{j-1} Y\right)
$$

is a $C_{s}^{\infty}$ vector field on $\tilde{M}$ if $Z=f_{*} Y-Y$ is of class $C_{s}^{\infty}$ on $M$. Lemma 2, applied to $\tilde{Y}$ and a smooth extension of $\hat{E}^{u}$ to $\tilde{M}$ gives the desired result.

The proof of Lemma $1^{\prime}$ is similar to that of Lemma 1 . The only place where the translation from the language of flows to diffeomorphisms is not obvious is at the proof of the sufficiency of (2.3) for the regularity of $Y^{s}$, so we give the details now.

Assuming that $Z^{s}$ is of class $C_{s}^{\infty}$, we consider the sequence of $C_{s}^{\infty}$ functions

$$
Y_{s}^{n}=-\sum_{j=1}^{n} \lambda_{j}^{s} \cdot\left(Z_{s} \circ f^{-j}\right)
$$

Differentiating along $X^{s}$, and manipulating as in the proof of Lemma 1,

$$
\begin{aligned}
X^{s} Y_{s}^{n}= & -\sum_{j=0}^{n}\left(X^{s} Z_{s}\right) \circ f^{-j}-\sum_{j=0}^{n}\left(X^{s} \lambda_{j}^{s}\right) \cdot\left(Z_{s} \circ f^{-j}\right) \\
= & -\sum_{j=0}^{n}\left[X^{s} Z_{s}-\left(X^{s} \lambda_{1}^{s}\right) \cdot\left(Y_{s} \circ f^{-1}\right)\right] \circ f^{-j} \\
& +\sum_{j=0}^{n} \sum_{k=0}^{\infty}\left[\left(X^{s} \lambda_{1}^{s}\right) \circ f^{-j}\right] \cdot\left(\lambda_{k}^{s} \circ f^{-j-1}\right) \cdot\left(Z_{s} \circ f^{-j-k-1}\right) \\
& -\sum_{j=0}^{n} \sum_{k=0}^{j-1}\left[\left(X^{s} \lambda_{1}^{s}\right) \circ f^{-k}\right] \cdot\left(\lambda_{j-k-1}^{s} \circ f^{-k-1}\right) \cdot\left(Z_{s} \circ f^{-j}\right),
\end{aligned}
$$

where we have used that $\lambda_{j}^{s}=\prod_{k=0}^{j-1}\left(\lambda_{1}^{s} \circ \mathrm{f}^{-k}\right)$ in order to compute its derivative along $X^{s}$, and that $Y_{s}=-\sum_{k=0}^{\infty} \lambda_{k}^{s} \cdot\left(Z_{s} \circ f^{-k}\right)$, since $Z=f_{*} Y-Y$.

Defining new indices $\tilde{j}=j, \tilde{k}=j+k$ in the first double sum, and interchanging the order of summation, we get two sums, one for $0 \leqq \tilde{j} \leqq \tilde{k} \leqq n-1$ and another for $0 \leqq \tilde{j} \leqq n \leqq \tilde{k}$. The first one cancels out with the last double sum in the previous 
expression, so we have

$$
\begin{aligned}
X^{s} Y_{s}^{n}= & -\sum_{j=0}^{n}\left[X^{s} Z_{s}-\left(X^{s} \lambda_{1}^{s}\right) \cdot\left(Y_{s} \circ f^{-1}\right)\right] \circ f^{-j} \\
& +\sum_{k=0}^{\infty} \sum_{j=0}^{n}\left[\left(X^{s} \lambda_{1}^{s}\right) \circ f^{-j}\right] \cdot\left(\lambda_{n+k-j}^{s} \circ f^{-j-1}\right) \cdot\left(Z_{s} \circ f^{-n-k-1}\right) .
\end{aligned}
$$

By (2.2) and Livsic's theorem (see [Li]), there exists a Hölder function $H$ on $M$ such that

$$
H \circ f-H=X^{s} Z_{s}-\left(X^{s} \lambda_{1}^{s}\right) \cdot\left(Y_{s} \circ f^{-1}\right) .
$$

Substituting this in the above expression, and taking into account that $\lambda_{n+k-j}^{s}=\lambda_{n-j}^{s} \cdot\left(\lambda_{k}^{s} f^{j-n}\right)$, we see that

$$
X^{s} Y_{s}^{n}=H-H \circ f^{n+1}+\sum_{j=0}^{n}\left[\left(X^{s} \lambda_{1}^{s}\right) \circ f^{-j}\right]\left(\lambda_{n-j}^{s} \circ f^{-j-1}\right) \cdot\left(Y_{s} \circ f^{-n-1}\right),
$$

and the absolute value of $X^{s} Y_{s}^{n}$ is bounded by a constant independent of $n$ as a consequence of (2.1). From this one gets that $Y_{s}=\lim _{n \rightarrow \infty} Y_{s}^{n}$ is uniformly Lipschitz on stable manifolds. The remaining part of the proof of Lemma $1^{\prime}$ is completely similar to that of Lemma 1. The details are left to the reader. As a consequence of Theorem 3 we have the following result on smooth conjugacy of Anosov flows in 3 dimensions.

Theorem 4. Let $X_{\tau},-a<\tau<a$, be a $C^{\infty}$ family of $C^{\infty}$ transitive Anosov vector fields on a compact 3-dimensional manifold. A necessary and sufficient condition for the existence of diffeomorphisms $g_{\tau},-a<\tau<a$, satisfying

$$
g_{\tau}^{*} X_{\tau}=X_{0}
$$

is that for any smooth family $\gamma_{\tau}$ of periodic orbits of $X_{\tau}$ both the periods and the Lyapunov exponents of $\gamma_{\tau}$ are independent of $\tau$. Moreover, in such a case the $g_{\tau}$ are a $C^{\infty}$ family of $C^{\infty}$ diffeomorphisms.

Remark. According to R. Mañé (talk at the workshop on Dynamical systems, CTIP, Trieste, June 1986) the periods of periodic orbits are by themselves a complete set of invariants for smooth conjugation of Anosov flows with $C^{1}$ invariant foliations (in particular geodesic flows). This is a consequence of a theorem by Feldman and Ornstein that gives $C^{1}$ conjugacy. A. Katok has announced a proof of the fact that the $C^{1}$ conjugacy implies $C^{\infty}$ conjugacy, based on the techniques of [LMM]. The techniques of [L1] also give a proof of this fact.

Proof of Theorem 4. The necessity is obvious. We prove the sufficiency of the conditions, so we assume the periods and Lyapunov exponents of corresponding orbits are the same. Differentiating (2.16) with respect to $\tau$ we see that it suffices to show that there exists a $C^{\infty}$ family of $C^{\infty}$ vector fields $H_{\tau}$ satisfying

$$
L_{X_{\tau}} H_{\tau}=\frac{d X_{\tau}}{d \tau}
$$


First we shall prove that there exists a continuous family of $C_{X_{\tau}}^{1}$ vector fields $H_{\tau}$ satisfying (2.17), and then we shall prove that $d X_{\tau} / d \tau$ satisfies the conditions of Theorem 3, so that the $H_{\tau}$ are actually of class $C^{\infty}$.

The existence of the $H_{\tau}$ is a consequence of the constancy of the periods, as we shall see now. By the version in [LMM, Appendix A], of Anosov's structural stability theorem, there is a $C^{\infty}$ isotopy $\tilde{h}_{\tau}$ and a $C^{\infty}$ family of $C^{\infty}$ functions $\eta_{\tau}$ such that

$$
\tilde{h}_{\tau}^{*} X_{\tau}=\eta_{\tau} X_{0}
$$

The same proof of Anosov's theorem gives that both $\eta_{\tau}$ and $\tilde{h}_{\tau}^{*} X_{\tau}$ and their successive derivatives with respect to $\tau$ are Hölder functions of order $\alpha$ for some fixed $\alpha>0$ depending only on $X_{\tau}$ (the only changes are in the functional setting, where one has to consider spaces of Hölder functions and sections of vector bundles; in the proof of Lemma A.7 one has to prove that all the functions and sections that appear are Hölder, but this is a consequence of the invariant subspaces of the flow being Hölder, see [Sh, Corollary 5.19]).

If we differentiate our last expression with respect to $\tau$ at $\tau=0$, taking into account that $\eta_{0}=1$ and $\widetilde{h}_{0}=\mathrm{Id}$, we see that

$$
L_{\tilde{H}_{0}} X_{0}+\left.\frac{d X_{\tau}}{d \tau}\right|_{\tau=0}=\left(\left.\frac{d \eta_{\tau}}{d \tau}\right|_{\tau=0}\right) \cdot X_{0}
$$

By the constancy of the periods of the periodic orbits, the integral of $\eta_{\tau}$ along each periodic orbit of $X_{0}$ is equal to the period of the orbit. This means that the average of $\eta_{\tau}-1$ along any periodic orbit of $X_{0}$ vanishes, so by Livsic's theorem, since $\eta_{\tau}-1$ and its successive derivatives with respect to $\tau$ are Hölder functions, there exists a $C^{\infty}$ family $\psi_{\tau}$ of Hölder functions such that $\eta_{\tau}-1=X_{0} \psi_{\tau}$. Then, by our last equality,

$$
L_{X_{0}} \tilde{H}_{0}=\left.\frac{d X_{\tau}}{d \tau}\right|_{\tau=0}-\left[X_{0}\left(\frac{d \psi_{\tau}}{d \tau}\right)\right] \cdot X_{0}=\left.\frac{d X_{\tau}}{d \tau}\right|_{\tau=0}-L_{X_{0}}\left[\frac{d \psi_{\tau}}{d \tau} \cdot X_{0}\right] .
$$

Since this can be done for any particular value of $\tau$ instead of $\tau=0$, our claim is proved. We shall see now that $d X_{\tau} / d \tau$ satisfies the conditions of Theorem 3 .

As a consequence of our hypothesis about the periods of periodic orbits, given any periodic orbit $\gamma$ of $X_{0}$ there exists a $C^{\infty}$ family of $C^{\infty}$ diffeomorphisms $h_{\tau}$ such that $\gamma$ is a periodic orbit of $h_{\tau}^{*} X_{\tau}$ for every $\tau$. By Theorem 1 in [F] applied to the flow defined by the family $X_{\tau}$ on $M \times \mathbb{R}$, we can make a further conjugation (we still call $h_{\tau}$ the resulting isotopy) in such a way that the previous property still holds and the local stable manifold of $\gamma$ with respect to $X_{\tau}^{1}=h_{\tau}^{*} X_{\tau}$ is independent of $\tau$.

We consider coordinates $(\theta, x) \in S^{1} \times I$ on these local stable manifolds in such a way that $X^{1 \tau}=T^{-1} \partial / \partial \theta$ on $S^{1} \times\{0\}$. We can also take them in such a way that $X_{0}^{1}=T^{-1} \partial / \partial \theta-T \lambda \partial / \partial x$, where $\lambda$ is the Lyapunov exponent of $\gamma$. This is a direct consequence of Sternberg's theorem, [St], on the linearization of contractive maps in the real line, which allows one to assume that the Poincare map of $X_{0}^{1}$ along the stable manifold of a point in $\gamma$ is linear. 
Assume now that on the stable manifold of $\gamma$,

$$
X_{\tau}^{1}=H_{\tau}(\theta, x) \frac{\partial}{\partial \theta}+V_{\tau}(\theta, x) \frac{\partial}{\partial x} .
$$

The Lyapunov exponents of $X_{\tau}^{1}$ along $\gamma$ are the same as those of

$$
\tilde{X}_{\tau}^{1}=\frac{\partial}{\partial \theta}+V_{\tau}(\theta, x) \cdot H_{\tau}(\theta, x)^{-1} \cdot \frac{\partial}{\partial x},
$$

which can be computed explicitly:

$$
\lambda_{\tau}=\left.\int_{0}^{1} \frac{\partial}{\partial x}\left[V_{\tau}(\theta, x) \cdot H_{\tau}(\theta, x)^{-1}\right]\right|_{x=0} d \theta .
$$

By the hypothesis about the Lyapunov exponents, the derivative with respect to $\tau$ of the above expression vanishes. Since

we see that

$$
V_{\tau}(\theta, 0)=H_{\tau}(\theta, 0)=\frac{\partial V_{\tau}}{\partial \tau}(\theta, 0)=\frac{\partial H_{\tau}}{\partial \tau}(\theta, 0)=0
$$

$$
\int_{0}^{1} \frac{\partial^{2} V_{\tau}}{\partial \tau \partial x}(\theta, 0) d \theta=0
$$

For $\tau=0, \partial / \partial x$ is a stable vector field, as a consequence of the explicit expression of $X_{0}$, and $\partial V_{\tau} / \partial \tau$ is the component of $d X_{\tau}^{1} / d \tau$ along the stable direction. Since the $C^{\infty}$ stable vector field $\partial / \partial x$ can be extended from the local stable manifold of $\gamma$ to a $C_{s}^{\infty}$ non-vanishing stable vector field on $M$, we see that $d X_{\tau}^{1} / d \tau$ satisfies (2.4) when $\tau=0$ for a particular stable section and, by the remark made after the statement of Theorem 4, it also satisfies (2.4) for any global non-vanishing stable vector field $X^{s}$. Since $X_{\tau}=g_{\tau}^{*} X_{\tau}^{1}$ with $g_{\tau}=h_{\tau}^{-1}$ and $g_{0}=\mathrm{Id}$,

$$
\left.\frac{d X_{\tau}}{d \tau}\right|_{\tau=0}=L_{G_{0}} X_{0}^{1}+\left.\frac{d X_{\tau}^{1}}{d \tau}\right|_{\tau=0}
$$

where $G_{\tau}$ is the vector field that generates the isotopy $g_{\tau}$. By Theorem $3, L_{G_{0}} X_{0}^{1}$ satiesfies (2.4), and this proves that $d X_{\tau} / d \tau$ also satiesfies (2.4) for $\tau=0$. By Theorem 3 again, the solution $H_{0}$ of (2.17) is of class $C^{\infty}$.

The above argument can be carried out for any $\tau$, since $\tau=0$ did not play any particular role. This finishes the proof of Theorem 4 , since the $C^{\infty}$ dependence on the parameter $\tau$ holds independently of any assumption on periods or Lyapunov exponents (see [LMM, Appendix A]).

Acknowledgements. We would like to thank R. Mañé, who told us about the lack of smoothness of the stable and unstable bundles of Anosov flows in 3 dimensions. A first version of this paper used the $C^{1}$ smoothness of those bundles in the proof. The second author also wants to thank J. Mather for useful indications concerning properties of cohomologous area preserving maps in $T^{2}$. 


\section{References}

[B] Banyaga, A.: Sur la structure du groupe des diffeomorphismes qui preservent une forme symplectique. Commun. Mat. Helv. 53, 174-227 (1978)

[C] Calabi, E.: On the group of automorphisms of a symplectic manifold. In: Problems in Analysis (symposium in honour of S. Bochner). Princeton, NJ : Princeton University Press 1970, pp. 1-26

[CEG] Collet, P., Epstein, H., Gallavotti, G.: Perturbations of geodesic flows on surfaces of constant negative curvature and their mixing properties. Commun. Math. Phys. 95, 61-112 (1984)

[CZ] Conley, C., Zehnder, E.: The Birkhoff-Lewis fixed point theorem and a conjecture of V.I. Arnold. Invent. Math. 73, 33-49 (1983)

[F] Fenichel, N.: Persistence and smoothness of invariant manifolds for flows. Indiana Univ. Math. J. 21, 3, 193-226 (1971)

[GK] Guillemin, V., Kazhdan, D.: On the cohomology of certain dynamical systems. Topology 19, 291-300 (1980)

[H] Herman, M.R.: Sur la conjugaison différentiable des diffeomorphismes du cercle à des rotations. Publ. Math. 49, 5-234 (1979)

[HP] Hirsch, M.W., Pugh, C.C.: Stable manifolds and hyperbolic sets. Proc. Symp. Pure Math. 14, AMS, 133-164 (1970)

[HPS] Hirsch, M.W., Pugh, C.C., Shub, M.: Invariant manifolds. Lecture Notes in Mathematics, Vol. 583. Berlin, Heidelberg, New York: Springer 1977

[L] Livsic, A.: Homology properties of $Y$-systems. Math. Notes 10, 754-757 (1971)

[L1] Llave, R. de la: Invariants for smooth conjugacy of hyperbolic dynamical systems, II. Commun. Math. Phys. 109, 369-378 (1987)

[LMM] Llave, R. de la, Marco, J.M., Moriyón, R.: Canonical perturbation theory of Anosov systems and regularity results for the Livsic cohomology equation. Ann. Math. 123, 537-611 (1986)

[M] Manning, A.: There are no new Anosov diffeomorphisms on tori. Am. J. Math. 96, (3), $422-429$ (1974)

[Ma] Mather, J.: Appendix to [S], ibid

[Mo] Moser, J.: On a theorem of Anosov. J. Differ. Equations 5, 411-440 (1969)

[MM] Marco, J.M., Moriyón, R.: Invariants for smooth conjugacy of hyperbolic dynamical systems, I. Commun. Math. Phys. 109, 681-689 (1987)

[P] Plante, J.F.: Anosov flows. Am. J. Math. 94, 729-754 (1972)

[S] Smale, S.: Differentiable dynamical systems. Bull. AMS 73, 747-817 (1967)

[Sh] Shub, M.: Stabilité globale des systèmes dynamiques. Asterisque 56, (1978)

[St] Sternberg, S. : On local $C^{r}$ contractions on the real line. Duke Math. J. 24, 97-102 (1957)

Communicated by J.N. Mather

Received August 22, 1986 
